\title{
Male rats prefer sex to food after 6 days of food deprivation*
}

\author{
BENJAMIN D. SACHS and EDWARD MARSAN \\ University of Connecticut, Storrs, Conn. 06268
}

Thirty male rats were deprived of food for 0-6 days, then presented simultaneously with food and a receptive female. Twenty-five males ejaculated twice within $30 \mathrm{~min}$; all but four of them deferred eating until after ejaculation. Food deprivation increased the latencies to begin copulation (depressed the sexual arousal mechanism) and reduced the stimulation needed for the first ejaculation (facilitated the copulatory and ejaculatory mechanism).

Suggestions that food deprivation reduces sexual motivation (Olds, $1958 \mathrm{a}$, b; Herberg, 1963) have received little experimental support. Jarmon \& Gerall (1961) reported that guinea pigs deprived of food for $51 \mathrm{~h}$ had normal copulatory activity. Sachs (1965) found that rats copulating after 1.9 days without food did have longer latencies to initiate copulation. However, no other measures of copulatory behavior were changed significantly.

A more direct test of the interactions between tendencies to eat and to copulate would involve a preference test. Stone \& Ferguson (1938) gave male rats a choice in a T-maze between food and a receptive female. The rats preferred food on $62 \%$ of the trials, but the results are difficult to interpret because of inadequate control over deprivation conditions and behavior in the goalboxes. Larsson (1956) trained food-deprived male rats to press a bar to obtain food pellets. When rats deprived of food for $23 \mathrm{~h}$ were presented with a receptive female in the Skinner box, they tended to defer both the operant and eating until after ejaculation.

In the present experiment, food and a receptive female were made freely and simultaneously available to male rats that had been deprived of food for 1-6 days.

\section{SUBJECTS}

Thirty hooded male rats (Long Evans from Blue Spruce Farms, Inc., Altamont, N.Y.), approximately 100 days old at the start of the experiment, were selected from a larger number on the basis of their performance in screening tests for sexual behavior. All males had been housed individually since their arrival

* Supported by Research Grant HD-04048 to B. D. Sachs, from the National Institute of Child Health and Human Development. The hormones were supplied through the courtesy of $P$. Periman of Schering Corp., Bloomfield, N.J. Reprints are available from B. D. Sachs, Department of Psychology U-20, University of Connecticut. Storrs, Conn. 06268. from the supplier several weeks earlier. APPARATUS

Animals were maintained in $10 \times 6$ $x 7$ in. steel cages with mesh floor and front and with external food hopper and water bottle.

The test cages, $20 \times 12 \times 12 \mathrm{in}$. aquaria with wood shavings covering the floor, were in a separate room, illuminated during the tests by two red $40-\mathrm{W}$ bulbs. Behavioral events were recorded on an Esterline-Angus event recorder activated by a pushbutton panel.

\section{PROCEDURE}

After the initial screening, each male was given 4 or 5 weekly normative tests so that his behavior might reach a more stable level. In the normative tests, after the male had been in the test cage for $10 \mathrm{~min}$, a receptive female, $20 \mathrm{~g}$ of Purina Lab Chow, and a water bottle were placed with him. Testing continued for 30 min or until the first intromission following the second ejaculation, whichever came first. Copulatory behavior, eating, and drinking were recorded throughout the test.

At least $24 \mathrm{~h}$ after each normative test, each male was deprived of food for $24 \mathrm{~h}$. Then he was placed into the test cage with food for $15 \mathrm{~min}$. Thus, each male experienced. both copulation and eating in the test cage.

Following the normative tests, six males were assigned randomly to each of five deprivation conditions: $0,1,2$, 4 , or 6 days without food. Water continued to be available for all Ss. Food deprivation was timed so that, in the final test, each male would have copulated 7 days earlier. Thus, the final test was the same as the normative tests, except that the males had differing periods of food deprivation prior to the final test.

All tests were conducted during the first $3 \mathrm{~h}$ of the dark phase of the cycle (14L:10D). Receptivity was induced in ovariectomized females by subcutaneous implantation of estradiol benzoate.

\section{RESULTS}

Copulation

Despite up to 6 days of food deprivation, all but two of the males achieved intromission and all but four males ejaculated. At least four of the six males in each group achieved two ejaculations. Table 1 indicates the parameters of copulatory behavior for tnose males continuing to two ejaculations.

Because of the large variability for the four animals in the group deprived for 6 days, separate analyses of variance for each variable determined the significance of the differences among the scores inclusive and exclusive of this group. Most of the following summary of results and the discussion are based upon the analysis excluding the group deprived for 6 days.

Food deprivation had significant effects on every variable measured, except the first postejaculatory interval and the number of intromissions in the second series. Males with longer periods of deprivation tended toward longer mount and intromission latencies, fewer intromissions in the first series, and a shorter ejaculation latency in the first series. The intervals between intromissions in both series and the ejaculation latency in the second series were also significantly affected by food deprivation, but not in a monotonic manner. For the second ejaculation latency and the intercopulatory interval of the second series, there was evidence of a $U$-shaped function with a minimum at 1 -day deprivation.

\section{Eating}

Of the males that copulated, only five ate prior to gaining intromission. Furthermore, they spent little time eating before copulating. Of these five males, the four that eventually ejaculated spent $156,64,12$, and 3 sec eating prior to their first intromission. The first two of these males had been deprived for 4 days, the second two for 6 days.

of the 19 deprived males that ejaculated twice, only five ate during their first copulatory series. The median time spent eating by these five during the first series was $20 \mathrm{sec}$, a small fraction of the ejaculation latency.

After the first ejaculation, 58\% $(11 / 19)$ of the deprived males ate ( $m$ dn duration $=118 \mathrm{sec}$ ), but, as noted earlier, they resumed copulation at the normal time. No males ate during the second ejaculatory series.

The four males that did not ejaculate during the final test did spend a substantial portion of the 30-min period eating and drinking: 455 and $1,016 \mathrm{sec}$ for the two males that copulated but did not ejaculate, 792 and $924 \mathrm{sec}$ for the two that did not copulate. 
Table 1

Effects of Food Deprivation on Parameters of Copulation (Means)*

\begin{tabular}{|c|c|c|c|c|c|c|c|c|c|c|c|}
\hline $\begin{array}{c}\text { Days } \\
\text { Deprived } \\
\text { (N=6 Per } \\
\text { Group) }\end{array}$ & \multicolumn{2}{|c|}{$\begin{array}{l}\text { Number of Males } \\
\text { Copulating to }\end{array}$} & $\begin{array}{c}\text { ML } \\
\text { (Sec) }\end{array}$ & $\begin{array}{c}\text { IL } \\
(\text { Sec })\end{array}$ & $\begin{array}{c}\text { Number } \\
\text { of } I_{1}\end{array}$ & $\begin{array}{l}\text { EL, } \\
\text { (Sec) }\end{array}$ & $\begin{array}{l}\mathrm{ICI} \\
(\mathrm{Sec})\end{array}$ & $\begin{array}{l}\mathrm{PEI}_{1} \\
(\mathrm{Sec})\end{array}$ & $\begin{array}{c}\text { Number } \\
\text { of } I_{2}\end{array}$ & $\begin{array}{c}E L_{2} \\
(\operatorname{Sec})\end{array}$ & $\begin{array}{l}\mathrm{ICI}_{2} \\
(\mathrm{Sec}) \\
\end{array}$ \\
\hline 0 & 6 & 6 & 8.8 & 32.7 & 13.8 & 321 & 32.4 & 266 & 5.2 & 210 & 36.5 \\
\hline 1 & 6 & 6 & 5.2 & 62.2 & 11.3 & 238 & 21.1 & 350 & 4.3 & 95 & 19.8 \\
\hline 2 & 5 & 5 & 36.6 & 62.0 & 11.4 & 230 & 43.7 & 329 & 5.6 & 132 & 23.2 \\
\hline 4 & 5 & 4 & 75.5 & 88.0 & 7.3 & 171 & 24.7 & 387 & 5.0 & 120 & 24.9 \\
\hline 6 & 4 & 4 & 99.8 & 123.0 & 8.3 & 255 & 29.6 & 391 & 5.0 & 152 & 32.3 \\
\hline Including & \multirow{2}{*}{\multicolumn{2}{|c|}{$\begin{array}{l}F(4,20 \text { df })= \\
P=\end{array}$}} & 12.05 & 3.22 & 3.26 & 1.42 & 2.11 & 1.00 & 0.51 & 1.23 & 2.05 \\
\hline Group 6 & & & $<.001$ & $<.05$ & $<.05$ & $>.20$ & $>.10$ & $>.20$ & $>.50$ & $>.20$ & $>.10$ \\
\hline $\begin{array}{c}\text { Excluding } \\
\text { Group } 6\end{array}$ & \multicolumn{2}{|c|}{$\begin{array}{l}F(3,17 d f)= \\
P=\end{array}$} & $\begin{array}{r}14.37 \\
<.001\end{array}$ & $\begin{array}{r}3.74 \\
<\quad .05\end{array}$ & $\begin{array}{r}4.97 \\
<.025\end{array}$ & $\begin{array}{r}6.36 \\
<\quad .005\end{array}$ & $\begin{array}{r}4.34 \\
<\quad 025\end{array}$ & $\begin{array}{r}0.71 \\
>\quad 20\end{array}$ & $\begin{array}{r}0.94 \\
>\quad 20\end{array}$ & 5.19 & 5.26 \\
\hline
\end{tabular}

*.HL = mount latency (time from presenting female to first mount); IL = intromission latency (time from presenting female to first intromission): No. $I=$ number of intromissions to ejaculation; $E L=$ ejaculation latency (time from first intromission to ejaculation); $I C I=$ intercopulatory interval (EL $\div$ No. I): $P E I=$ postejaculatory interval (time from ejaculation to next intromission). Subscript numbers refer to ejaculatory series. All data are computed only for those males copulating to two ejaculations. Analyses of variance are based upon reciprocally transformed scores. except for No. I and ICI.

\section{DISCUSSION}

The hypothesis (Olds, 1958a, b; Herberg, 1963) that food deprivation reduces sexual motivation leads to the predictions that (1) with increased deprivation, fewer males should copulate, (2) those males that do copulate should show reduced sexual arousability, and (3) more males should prefer food to sex. The data obtained offer only very limited confirmation of these predictions.

Concerning the first prediction, in this experiment, as in that of Sachs (1965), longer periods of deprivation were associated with a reduction in the number of males copulating. However, even with 6 days of food deprivation, four of six males did copulate to two ejaculations within $30 \mathrm{~min}$.

Consistent with the second prediction and with the data of Sachs (1965) was the observed increase in latencies to begin copulating. However, analysis of the changes in copulatory behavior of the food-deprived male rats reveals that the effects of deprivation on sexual arousal are not simple. Sexual arousability, as measured by the mount and intromission latencies, is apparently reduced, but rearousal following ejaculation (PEI) is unaffected. In addition, it appears from the present data that once copulation is initiated, arousal to ejaculation is actually facilitated by food deprivation. Males with longer periods of deprivation had fewer intromissions to ejaculation (in the first series) and shorter ejaculation latencies. Thus, food deprivation seems to act to increase the stimulation needed to initiate copulation and to reduce the stimulation needed to achieve ejaculation. Expressed in the terms of Beach's (1956) two-factor theory, food deprivation seems to depress the sexual arousal mechanism (SAM) and to facilitate the copulatory and ejaculatory mechanism (CEM) or, alternatively, to raise the copulatory threshold and lower the ejaculatory threshold.

The fact that deprivation had no significant effect on postejaculatory interval and effected an acceleration of the intercopulatory interval indicates that inanition and fatigue played little or no role in the animals' behavior. However, the variability among the males deprived for 6 days suggests that, with longer deprivation periods, these variables might come to play an important role.

The third prediction, that food deprivation should lead to a preference for food over sex, was also confirmed only to a very limited extent. Only 5 of the 24 deprived males ate before copulating and only for a brief period. Only 2 of the deprived males ate to the exclusion of sexual behavior during the 30 -min test.

The copulatory behavior of male rats consists of bouts of mounting the females (Sachs \& Barfield, 1970), separated by intervals of grooming and other "nonsexual" activities (Dewsbury, 1967). In principle, males could eat before initiating copulation and between mount bouts, so that feeding and copulation could occur alternately. In fact, this alternation in activities of food-deprived rats was not observed. Rather, males tended to copulate to ejaculation with little or no eating prior to ejaculation.

The effect of food deprivation in delaying the initiation of copulation bears further comment. Hinde (1970, p. 203) interpreted the longer latencies of food-deprived rats ". . . in terms of priorities and inhibiting effects: in the food-deprived animal food-searching behavior is prepotent and sexual behavior suppressed [Sachs, 1965]." The results of the present experiment cast doubt upon the second part of this interpretation, since most deprived males did not eat prior to copulation but still displayed longer mount and intromission latencies.

The inhibition of food-related behavior by sex-related behavior has been described before. Gantt (1950) worked with restrained dogs and cats in a conditioned reflex paradigm, using penile stimulation as a US for erection and food as a US for salivation and chewing. He concluded: "Although many physiological functions are reciprocally inhibitory, when sexual - excitation is in the ascendancy, it appears to be the most exclusively imperative, excepting perhaps immediate threats to life. Even in our laboratory studies with artificial sexual stimuli, sexual conditional reflexes (crs) were not abolished after five days starvation [Gantt, 1950, p. 1031]." Gantt $(1950$, p. 1050) also observed that "the male dog, even though hungry, and the cat, too, refuses to take food during sexual excitation whereas the female does not refuse food then." 1

Larsson (1956) engaged in a thorough exploration of the interactions between sexual and food-oriented behaviors. His general procedure was to train male rats to press a bar in order to obtain food and then to test the effect on the operant of various conditions of food and sex deprivation. In general, he found that males would not press the bar for food once they initiated a copulatory series. Rather, they would wait until some time after ejaculation before pressing the bar for food. On those occasions that the male pressed the bar accidentally during a series, he rarely ate the pellet that was delivered.

In concordance with Hinde (1970), we may view the male's response tendencies as being hierarchically organized but with sexual behavior having normal predominance over 
feeding and being incompatible with it. The initiation of one of these activities tends to suppress the other. In the context of Beach's (1956) two-factor theory, activation of the SAM or the CEM inhibits the tendency to eat. Discharge of the CEM by ejaculation releases inhibition, permitting the food-deprived male to eat until the SAM is again aroused sufficiently to reimpose inhibition on eating.

\section{REFERENCES}

BEACH, F. A. Characteristics of masculine "sex drive." In M. R. Jones (Ed.). Nebraska symposium on motivation. Lincoln: University of Nebraska Press,
1956. Pp. 1-32.

DEWSBURY, D. A. A quantitative description of the behavior of rats during copulation. Behaviour, 1967, 29. 154-178.

GANTT, W. H. Disturbances in sexual functions during periods of stress. In Life stress and bodily disease. Research Publications of the Association for Publications of the Association for
Research in Nervous \& Mental Diseases. $1950,29,1030-1050$.

HERBERG, L J Seminal ejaculation following positively reinforcing electrical stimulation of the rat hypothalamus. Journal of Comparative \& Physiological Psychology, 1963, 56, 679-685.

HINDE, R. A Animal behaviour. New York: McGraw-Hill, 1970.

JARMON, H., \& GERALL, A. A. The effect of food deprivation upon the sexual performance of male guinea pigs. Journal of Comparative \& Physiological Psychology, 1961, 54, 306-309.

LARSSON, $\dot{K}$. Conditioning and sexual behavior in the male albino rat. Acta Psychologica Gothoburgensia, 1956. 1 , 1-269. (Stockholm: Almquist \& Wiksell).

OLDS. J. Discussion. In G. E. W.

Wolstenholme and C. M. O'Connor (Eds.), Ciba Foundation symposium: Neurological basis of behavior. Boston: Little, Brown, 1958a. P. 219.

OLDS, J. Effects of hunger and male sex hormone on self-stimulation of the brain. Journal of Comparative \& Physiological Psychology, 1958b, 51, 320-324.

SACHS, B. D. Sexual behavior of male rats after one to nine days without food. Journal of Comparative \& Physiological Psychology, 1965, 60, 144-146.

SACHS, B. D., \& BARFIELD, R. J. Temporal patterning of sexual behavior in the male rat. Journal of Comparative \& Physiological Psychology, 1970, 73, 359-364.

$$
\text { NOTE }
$$

1. I am indebted to Larsson (1956) for calling these observations to $\mathrm{my}$ attention. 